\title{
Motivação dos alunos para a utilização da tecnologia wiki: um estudo prático no ensino superior
}

Carolina Costal

Helena Alvelos'

Leonor Teixeira'

\section{Resumo}

No âmbito do processo ensino-aprendizagem (EA), os wikis permitem ajudar na criação de um ambiente dinâmico e colaborativo de aprendizagem, por meio da comunicação, troca de ideias e partilha de conhecimento. 0 presente trabalho faz parte de um projeto cujo intuito é avaliar a aceitação do uso dessa tecnologia como meio de promoção da qualidade do processo EA. Em particular, o estudo descrito neste artigo tem como objetivo avaliar a motivação para a utilização de wikis por parte de um grupo de alunos da área tecnológica do ensino superior e compará-la entre os alunos que pretendem continuar fazendo uso da ferramenta em atividades futuras e os que não planejam usar a tecnologia. Essa experiência foi feita em contexto de sala de aula e os dados foram obtidos através da aplicação de dois questionários e da análise do histórico do wiki criado para a atividade desenvolvida em classe. Os resultados revelaram que os alunos, apesar de conhecerem a ferramenta, desconheciam, na prática, o seu modo de edição. As características dos wikis mais bem classificadas pelos alunos se relacionam com a facilidade de utilização percebida e com a utilidade da ferramenta, na medida que em promove a aprendizagem por meio de conteúdos colocados pelos outros e a realização de trabalhos de grupo sem depender da presença física nem dos horários dos seus elementos. Verificou-se, ainda, que a motivação para o uso dos wikis revelou-se, em alguns itens, significativamente diferente entre os grupos comparados.

\section{Palavras-chave}

Wikis - Processo ensino-aprendizagem - Ensino superior Modelo TAM.

I- Universidade de Aveiro, Aveiro, Portugal.

Contatos: carolinacosta@ua.pt;

Iteixeira@ua.pt;

helena.alvelos@ua.pt 


\title{
The students' motivation to use the wiki technology: a practical study in higher education
}

Carolina Costa'

Helena Alvelos'

Leonor Teixeiral

\begin{abstract}
Within the teaching-learning (TL) process, the wikis help to create a dynamic and collaborative learning environment through communication, exchange of ideas and sharing of knowledge. The present work is part of a project whose aim is to evaluate the acceptance of the use of this technology as a means to promote the quality of the TL process. In particular, the study described in this article has as its objective to assess the motivation for the use of the wikis by a group of students from the technological area of higher education, and to carry out a comparison between students that intend to continue making use of this tool in future activities and those that do not plan to use this technology. This experience was made in the context of a classroom, and the data were obtained through the application of two questionnaires and analysis of the history of the wiki created for the activity developed in the classroom. The results showed that the students, despite knowing the tool, did not know in practice about its editing mode. The features of the wikis better classified by the students are related to the perceived ease of use and with the usefulness of the tool, in so far as it promotes learning through contents placed in it by others and through the conduction of group work relying neither on the physical presence of the members nor on their schedules. It has also been observed that the motivation for the use of the wikis turned out to be, in some items, significantly different among the groups compared.
\end{abstract}

\section{Keywords}

Wikis - Teaching-learning process - Higher education - TAM model.

I- Universidade de Aveiro, Aveiro, Portugal. Contacts: carolinacosta@ua.pt;

Iteixeira@ua.pt;

helena.alvelos@ua.pt 
As competências que os alunos devem alcançar na sua aprendizagem podem ser melhoradas ou facilitadas por meio de métodos pedagógicos que utilizam novas Tecnologias da Informação e Comunicação (TICs). No entanto, quando se pretende utilizar qualquer tecnologia no processo ensino-aprendizagem, o professor deve ter em conta a sua integração em uma perspetiva pedagógica para que esse uso seja o mais adequado possível.

0 wiki é considerado uma tecnologia útil no processo EA, permitindo ajudar a criação de um ambiente dinâmico e colaborativo por meio da comunicação, troca de ideias e partilha de conhecimento. A aceitação, em geral, de tecnologias, é normalmente avaliada através de modelos teóricos como, por exemplo, o Technology Acceptance Model (TAM).

0 presente trabalho faz parte de um projeto que tem como objetivo avaliar a aceitação da tecnologia wiki como meio de melhorar a qualidade do processo EA. Em termos metodológicos, este trabalho foi dividido em três fases e os dados foram obtidos a partir da aplicação de dois questionários e da análise do histórico do wiki, criado no contexto da atividade realizada com os alunos. Em particular, este estudo tem como objetivo avaliar a motivação para o uso de wikis de um grupo de alunos de uma Unidade Curricular (UC) da área tecnológica do $2^{\circ}$ ciclo da Universidade de Aveiro (UA), de modo a compará-la entre os alunos que pretendem e os que não pretende continuar a utilizar a tecnologia em atividades futuras.

Este trabalho está estruturado em cinco seções. A primeira descreve brevemente o modelo TAM, um dos mais utilizados para avaliar a motivação para o uso de tecnologias. A segunda aborda o papel dos wikis no processo EA e apresenta algumas atividades em que se fez uso dessa ferramenta em contexto educacional. A terceira seção, por sua vez, descreve a metodologia adotada no estudo prático. A quarta faz a análise e discussão dos principais resultados relativos à motivação para a utilização de wikis na aprendizagem. Por fim, apresentam-se as conclusões alcançadas nesta investigação.

\section{Motivação dos utilizadores para o uso de tecnologias}

Existem diversos modelos teóricos que avaliam a aceitação de tecnologias por parte dos utilizadores das diversas ferramentas, tais como o e-mail (DAVIS; BAGOZZI; WARSHAW, 1989), a Web 2.0 (MEI-YING et al., 2008; HUANG; YOO, 2010; UÇRICH; KARVONEN, 2011), as redes sociais (KWON; WEN, 2010), o YouTube (YANG et al., 2010) ou os wikis (WESLEY; YU-HAO, 2011).

Neste contexto, um dos modelos mais utilizados é TAM, desenvolvido por Davis (1986) e que, por sua vez, serviu de base para o desenvolvimento de outros, como, por exemplo, o modelo da Teoria Unificada de Aceitação e Uso de Tecnologia - Unified Theory of Acceptance and Use of Technology (UTAUT) (VENKATESH et al., 2003).

Segundo o modelo TAM, a motivação dos utilizadores para o uso de tecnologias é determinada por três variáveis: facilidade de utilização percebida - Perceived Ease of Use (PEOU), utilidade percebida - Perceived Usefulness (PU) e a atitude para utilizar - Attitude Toward Using (ATU). Essas variáveis, por sua vez, são influenciadas por outras variáveis externas $\left(\mathrm{X}_{1}, \mathrm{X}_{2} \mathrm{e}\right.$ $\mathrm{X}_{3}$ ) e contribuem para a utilização do sistema - Actual System Use (ASU) (DAVIS, 1986), tal como se encontra esquematizado na figura 1.

A facilidade de utilização percebida (PEOU) é definida como o grau em que um indivíduo acredita que o uso de um determinado sistema é intuitivo, não exigindo grande esforço (DAVIS, 1986; 1989), podendo ser influenciado por variáveis externas.

A utilidade percebida (PU) é definida como o grau em que um indivíduo acredita que a utilização do sistema contribui para aumentar o desempenho do seu trabalho (DAVIS; BAGOZZI; WARSHAW, 1989). Pode ser influenciada 
Figura 1 - Modelo TAM adaptado de Davis (1986)

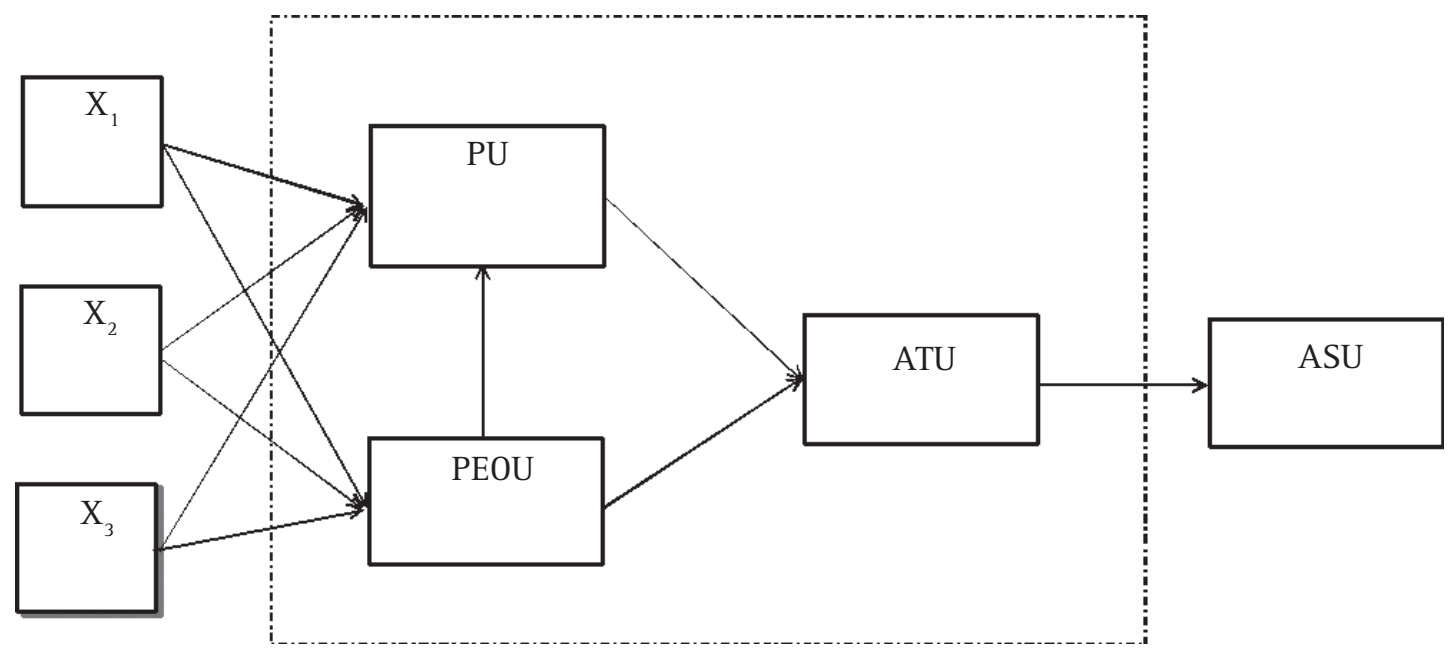

Fonte: Dados da pesquisa.

por variáveis externas e pela PEOU, dado que tecnologias percebidas como mais fáceis de usar tendem a ser vistas como mais úteis.

A atitude para utilizar (ATU) é definida como um sentimento positivo ou negativo de um indivíduo sobre a utilização do sistema (DAVIS, 1986; 1989; DAVIS; BAGOZZI et al., 1989) e é influenciada pelas duas variáveis anteriores.

Finalmente, o segundo o modelo, a utilização da tecnologia (ASU), depende da motivação do indivíduo para a utilizar a ferramenta através da sua atitude, expressa pela variável ATU.

\section{Wikis no processo ensino- aprendizagem}

0 wiki é uma tecnologia Web 2.0 que possibilita a construção de repositórios de conhecimento baseado num conjunto de páginas Web interligadas (FRANKLIN; HARMELEN, 2007; GROSSECK, 2009; KEAR et al., 2010), podendo ser visualizadas, criadas, editadas e modificadas por qualquer utilizador através de um editor de texto (DE PEDRO et al., 2006; KEAR et al., 2010). Esse conceito foi criado por Ward Cunningham em 1995 (ZEINSTEJER, 2008) e a sua filosofia consiste em manter um espaço na Web completamente aberto, onde qualquer utilizador pode modificar, estruturar e organizar documentos de diversas formas (KEAR et al., 2010).

Para além da funcionalidade de edição colaborativa em uma mesma página, um wiki apresenta normalmente uma área onde os participantes podem discutir o seu conteúdo atual. Esse espaço de discussão pode igualmente ser utilizado pelos participantes para testar as suas ideias (PIFFARÉ; STAARMAN, 2011). Existe também a opção de visualizar o histórico do wiki, com a capacidade de recuperar conteúdos que tenham sido excluídos anteriormente ou acidentalmente pelos participantes (LAI; NG, 2011).

No processo ensino-aprendizagem, os wikis permitem ajudar na criação de um ambiente dinâmico e colaborativo de aprendizagem, através da discussão aberta e da troca de ideias e de opiniões, no qual os alunos podem ter uma participação ativa na criação do conhecimento (MEYER, 2010; SU; BEAUMONT, 2010; LAI; NG, 2011). Os wikis proporcionam novas formas de aprendizagem em uma lógica democrática e centrada no aluno (KEAR et al., 2010). Dessa forma, é considerado como uma ferramenta útil no processo EA, promovendo a escrita e a aprendizagem colaborativa, ao mesmo tempo em que potencializa a criatividade de cada utilizador (CRESS; KIMMERLE, 2008). 
Quando o professor faz uso de atividades com wikis, pode promover uma nova forma de ensino, sendo uma alternativa ou complemento aos métodos e ferramentas de aprendizagem tradicionais (LAI; NG, 2011). Diferentes autores (RAITMAN; AUGAR; ZHOU, 2005; DE PEDRO et al., 2006; WAN; ZHAO, 2007; CHU, 2008; GRACE, 2009; GROSSECK, 2009; ONER, 2009; KEAR et al., 2010; MEYER, 2010; LAI; NG, 2011) têm estudado o impacto da utilização da ferramenta no processo EA, tendo, alguns deles, contribuído para a identificação de um conjunto de vantagens e desvantagens.

Algumas das vantagens da utilização de wikis em contexto EA são: (i) permitir a colaboração conjunta e promover a partilha de conteúdos (GROSSECK, 2009; KEAR et al., 2010); (ii) controlar o acesso aos recursos através da autenticação dos utilizadores (GROSSECK, 2009; KEAR et al., 2010); (iii) possibilitar a avaliação da participação individual de cada utilizador (DE PEDRO et al., 2006; CHU, 2008); (iv) possibilitar a visualização das alterações introduzidas e a recuperação de conteúdo removido ou modificado (DE PEDRO et al., 2006), e (iv) permitir o acesso ao histórico dos conteúdos (quem colocou o quê e quando) (DE PEDRO et al., 2006).

As desvantagens da utilização de wikis referidas por alguns autores são: (i) ser, por vezes, difícil a compreensão dos conteúdos editados por outras pessoas (LAI; NG, 2011); (ii) ser necessário conhecer as ferramentas de edição de texto (DE PEDRO et al., 2006; CHU, 2008; KEAR et al., 2010); (iii) existir o receio de que outras pessoas vejam o trabalho que ainda não está acabado (DE PEDRO et al., 2006), e (iv) ser, por vezes, difícil de avaliar a qualidade de alguns conteúdos (GROSSECK, 2009).

Para superar algumas das limitações apresentadas, deve-se considerar que a utilização de wikis no processo EA depende, em grande parte, dos professores e do seu conhecimento sobre a utilização e a organização das atividades com a ferramenta. Note-se que a ferramenta é, normalmente, mais utilizada pelos alunos para visualizar conteúdos (COSTA; TEIXEIRA; ALVELOS, 2011), estando, no entanto, o seu maior potencial na componente de edição.

Os wikis podem ser utilizados pelos intervenientes no processo EA, nomeadamente alunos e professores, no sentido de apoiar determinadas atividades pedagógicas. Alguns autores têm analisado essas atividades, que se descrevem, em seguida, resumidamente.

- A análise bibliográfica pode ser utilizada quando se pretende editar resumos de reflexões a respeito das leituras efetuadas (DUFFY; BRUNS, 2006);

- A avaliação pode ser efetuada com base em atividades desenvolvidas nos wikis (DUFFY; BRUNS, 2006; KANE; FICHMAN, 2009);

- 0 brainstorming pode ser aplicado para criar projetos em que os alunos são convidados a acrescentar artigos ou opiniões (GOKCEARSLAN; OZCAN, 2011), permitindo, assim, a criação de uma rede encadeada de recursos acerca de determinados assuntos (DUFFY; BRUNS, 2006);

- A atividade de comunicação traduz-se na publicação de recursos que poderão ser editados e comentados diretamente no documento, sendo acessível a todos (DUFFY; BRUNS, 2006);

- Os portfólios podem ser criados para a construção de um repositório de projetos pessoais ou de grupo (IT-USER SERVICES, 2008; GOKCEARSLAN; OZCAN, 2011), em que os alunos possam apresentar conteúdos e revê-los posteriormente (DUFFY; BRUNS, 2006);

- A listagem de links, para além de organizar a informação, permite criar listagens de ficheiros dos quais foram feitos uploads (IT-USER SERVICES, 2008; KANE; FICHMAN, 2009; GOKCEARSLAN; OZCAN, 2011);

- Os mapas de conceitos podem ser utilizados para criar dicionários de termos (DUFFY; BRUNS, 2006); 
- A organização da informação através dos wikis permite sumariar os conteúdos no topo da página, facilitando a navegação e a categorização dos conteúdos (IT-USER SERVICES, 2008; KANE; FICHMAN, 2009; GOKCEARSLAN; OZCAN, 2011);

- A atividade de pesquisa permite aos alunos desenvolverem projetos que envolvam pesquisas (DUFFY; BRUNS, 2006);

- A atividade de trabalho de grupo com utilização de wikis permite desenvolver trabalho em conjunto, podendo, os vários elementos, editar um único documento (DUFFY; BRUNS, 2006).

No planeamento de uma atividade deve se ter em conta alguns aspetos para a obtenção de resultados favoráveis ao processo EA. No quadro 1, apresentam-se seis aspetos para orientar o professor no planejamento de atividades com a ferramenta, segundo Foord (2007) e Gokcearslan e Ozcan (2011).

Quadro 1 - Linhas orientadoras para o planejamento de atividades com wikis (FOORD, 2007; GOKCEARSLAN; OZCAN, 2011)

\begin{tabular}{|c|c|}
\hline $\begin{array}{l}\text { Especificação do } \\
\text { objetivo geral }\end{array}$ & $\begin{array}{l}\text { - } 0 \text { objetivo deve ser claro, simples e autoexplicativo, devendo ser explicitados os benefícios em contribuir para a edição } \\
\text { do wiki ou para o produto final; } \\
\text { - } 0 \text { objetivo deve ser compreendido por todos; } \\
\text { - } 0 \text { wiki deve apresentar os objetivos específicos para os quais foi criado; } \\
\text { - Os alunos devem estar informados quando o trabalho no wiki ser alvo de avaliação; }\end{array}$ \\
\hline $\begin{array}{l}\text { Definição de } \\
\text { prazos }\end{array}$ & $\begin{array}{l}\text { - } 0 \text { tempo das diferentes fases de utilização deve ser definido; } \\
\text { - } 0 \text { prazo final deve ser estabelecido; }\end{array}$ \\
\hline Propriedade & $\begin{array}{l}\text { - } 0 \text { espaço deve ser adequado aos participantes, de forma a fomentar a competição entre os grupos, promovendo um } \\
\text { desafio para todos, fazendo com que todos possam apropriar-se da ferramenta; }\end{array}$ \\
\hline $\begin{array}{l}\text { Restrição do } \\
\text { objetivo }\end{array}$ & $\begin{array}{l}\text { - A estrutura do wiki deve ser a que se pretende dar à página; } \\
\text { - Devem ser criados pontos de partida para que a edição do wiki, os quais devem estar ligados à estrutura da ferramenta; }\end{array}$ \\
\hline $\begin{array}{l}\text { Regras de } \\
\text { compromisso }\end{array}$ & $\begin{array}{l}\text { - Deve-se reforçar quem pode editar o wiki; } \\
\text { - As orientações devem ser claras e estabelecidas para que os alunos saibam que partes podem editar; } \\
\text { - As orientações podem fornecer indicações sobre a utilização aceitável e inaceitável; }\end{array}$ \\
\hline Navegação & $\begin{array}{l}\text { - } 0 \text { moderador do wiki pode criar a estrutura para os participantes seguirem; } \\
\text { - A navegação deve ser clara e simples, não devendo ser necessários muitos passos para se encontrar uma parte } \\
\text { específica do wiki. }\end{array}$ \\
\hline
\end{tabular}

Fonte: Dados da pesquisa.

São vários os estudos que descrevem a utilização de wikis no contexto EA. Alguns desses têm com objetivos de:

- Analisar as reflexões dos alunos a respeito de suas experiências de colaboração em wikis (RAITMAN; AUGAR; ZHOU, 2005);

- Analisar e avaliar os aspetos essenciais para o êxito da implementação de um wiki (SU; BEAUMONT, 2010);

- Identificar e compreender os fatores que influenciam a utilização, a utilidade e a intenção do uso de wikis no futuro (GUO; STEVENS, 2011);

- Partilhar e descrever experiências com a utilização de wikis (COLE, 2009; ONER, 2009);
- Investigar a facilidade de comunicação baseada numa discussão estruturada e a sua relação com a participação no wiki (WICHMANN, 2012);

- Explorar as potenciais utilizações (CHAO, 2007), formas colaborativas (COUTINHO; JUNIOR, 2007; FORTE; BRUCKMAN, 2007) e formas participativas utilizando a tecnologia wiki (COUTINHO; JUNIOR, 2007);

- Proporcionar uma framework para o processo EA baseada na utilização de wikis (CUBRIC, 2007).

Os principais resultados dos referidos estudos são apresentados no quadro 2 . 
Quadro 2 - Principais resultados de estudos relativos à utilização de wikis no contexto EA

\begin{tabular}{|c|c|}
\hline Resultados & Autores \\
\hline - os wikis são fáceis de utilizar; & $\begin{array}{l}\text { Raitman, Augar, Zhou (2005); Chao } \\
\text { (2007); Coutinho e Junior (2007; Cubric } \\
\text { (2007); Su e Beaumont (2010) }\end{array}$ \\
\hline - o uso do wiki permitiu fazer trabalhos de grupo de forma mais fácil; & Oner (2009) \\
\hline - a atividade com wikis ajudou na aprendizagem; & Coutinho e Junior (2007); Cubric (2007) \\
\hline $\begin{array}{l}\text { - a utilização do wiki facilitou a partilha de ideias (independentemente do horário e local), levando } \\
\text { o aluno a poder desempenhar um papel mais ativo nos trabalhos de grupo, uma vez que toda a } \\
\text { comunicação e contribuições são visíveis para todos e as opiniões dos colegas podem melhorar } \\
\text { a qualidade geral do trabalho; }\end{array}$ & Cubric (2007); Oner (2009) \\
\hline $\begin{array}{l}\text { - o nível de utilização de wikis é influenciado pelo nível de experiência e conhecimento prévios } \\
\text { dos alunos; }\end{array}$ & Guo e Steven (2011) \\
\hline $\begin{array}{l}\text { - a utilização de wikis possibilita a visualização das mensagens e dos trabalhos dos colegas, } \\
\text { permitindo adquirir novos conhecimentos; }\end{array}$ & Coutinho e Junior (2007); Oner (2009) \\
\hline - o wiki é uma boa ferramenta para colaboração em projetos; & Chao (2007) \\
\hline - os alunos preferem participar em atividades pré-definidas; & Cole (2009) \\
\hline $\begin{array}{l}\text { - os comentários do professor às contribuições dos alunos é um fator de motivação para a } \\
\text { participação na atividade com o wiki, melhorando a qualidade do trabalho; }\end{array}$ & Coutinho e Junior (2007); Cubric (2007) \\
\hline - existe a necessidade de um apoio adicional para a edição dos wikis pelos alunos; & Wichmann (2012) \\
\hline $\begin{array}{l}\text { - a utilização de wikis levantam diversas preocupações sobre a possibilidade de plágio e } \\
\text { vandalismo de conteúdos; }\end{array}$ & Oner (2009) \\
\hline - os fatores culturais e institucionais influenciam a adoção de wikis na sala de aula; & Forte e Bruckman, (2007) \\
\hline $\begin{array}{l}\text { - as experiências educacionais devem preparar os alunos para se tornarem participantes } \\
\text { cuidadosos, críticos e competentes nas atividades de construção de conhecimento; }\end{array}$ & Forte e Brickman (2007) \\
\hline - os alunos sentem algum desconforto na utilização síncrona/simultânea de seções dos wikis; & Raitman, Augar, Zhou (2005) \\
\hline - a disponibilidade de um fórum de discussão permite uma maior contribuição para o wiki; & Wichmann (2012) \\
\hline - os alunos pretendem/recomendam utilizar wikis em projetos futuros. & $\begin{array}{l}\text { Chao (2007); Cubric (2007); Guo e } \\
\text { Stevens (2011) }\end{array}$ \\
\hline
\end{tabular}

Fonte: Dados da pesquisa.

\section{Metodologia}

Este trabalho insere-se no âmbito de um projeto que tem como objetivo avaliar a aceitação da tecnologia wiki como meio de melhorar a qualidade do processo EA, tendo sido levado a cabo em uma Unidade Curricular (UC) de Tecnologias Aplicadas à Gestão de Informação (TAGI), lecionada ao $2^{\circ}$ ano da licenciatura em Engenharia e Gestão Industrial (EGI) da Universidade de Aveiro (UA) e frequentada por cerca de 60 alunos distribuídos em quatro turmas.

0 projeto foi estruturado em três fases. $\mathrm{Na}$ primeira, pretendeu-se perceber o nível de utilização prévia e a finalidade de utilização de wikis por parte dos alunos, por meio da aplicação de um questionário, em formato papel, aos estudantes que frequentavam a UC.

Na segunda fase, objetivou-se avaliar a participação dos alunos em uma experiência com a edição de um wiki (wiki TAGI), criado especificamente para essa atividade. Dado que parte da avaliação da UC consistia em fazer um trabalho de grupo, o wiki TAGI foi utilizado para os alunos comunicarem aos demais colegas e ao docente da UC a constituição dos grupos e o tema escolhido para o trabalho. Nesse contexto, pretendia-se que os alunos, através 
do $w i k i$, discutissem e partilhassem ideias para o trabalho a ser desenvolvido, indicassem as principais funcionalidades que pretendiam implementar, referissem as expetativas que tinham e especificassem os objetivos que pretendiam atingir. A participação dos alunos na realização dessa atividade foi analisada com base no histórico do wiki TAGI.

Por último, na terceira fase, pretendeu-se avaliar a motivação dos alunos para a utilização de wikis no processo EA após a experiência, recorrendo-se a um segundo questionário no qual foram colocadas questões relativas às três variáveis do modelo TAM, referidas anteriormente, e avaliadas em uma escala de 1 (Não concordo nada) a 5 (Concordo totalmente). A variável facilidade de utilização percebida (PEOU) representa o grau com o qual os alunos acreditam que a utilização de wikis é intuitiva, não exigindo grande esforço, enquanto que a utilidade percebida (PU) representa o grau com que os alunos acreditam que a utilização de wikis lhes é útil. A atitude para utilizar (ATU) representa os sentimentos positivos ou negativos dos alunos relativamente à utilização de wikis no contexto de aprendizagem. No quadro 3, apresentam-se as questões que foram colocadas aos alunos relativamente a cada uma das variáveis.

Quadro 3 - Questões colocadas aos alunos para avaliar a motivação para a utilização de wikis no processo EA

\begin{tabular}{|c|c|}
\hline Variável & Item \\
\hline $\begin{array}{l}\text { Facilidade } \\
\text { de utilização } \\
\text { percebida (PEOU) }\end{array}$ & $\begin{array}{l}\text { PEOU1 - É necessário consultar frequentemente o guião de apoio/ícone de ajuda para utilizar os wikis. } \\
\text { PEOU2 - Os ícones e o texto associados aos wikis são de fácil compreensão. } \\
\text { PEOU3 - É fácil lembrar-me de como executar as tarefas relacionadas com a edição dos wikis. } \\
\text { PEOU4 - Fico confuso quando utilizo um wiki. } \\
\text { PEOU5 - Cometo erros frequentemente quando utilizo um wiki. } \\
\text { PEOU6 - Aprender a utilizar os wikis é fácil. } \\
\text { PEOU7 - No geral, acho que os wikis são fáceis de utilizar. }\end{array}$ \\
\hline $\begin{array}{l}\text { Utilidade percebida } \\
\text { (PU) }\end{array}$ & $\begin{array}{l}\text { PU1 - A utilização de wikis melhora o resultado da minha aprendizagem. } \\
\text { PU2 - A utilização de wikis permite-me organizar melhor o meu trabalho. } \\
\text { PU3 - A utilização de wikis faz-me poupar tempo. } \\
\text { PU4 - A comunicação proporcionada pelos wikis permite-me aprender através dos conteúdos colocados pelos outros. } \\
\text { PU6 - Os wikis permitem fazer trabalhos de grupo sem depender da presença física dos outros elementos do grupo. } \\
\text { PU7 - No geral, acho os wikis úteis para a minha aprendizagem. }\end{array}$ \\
\hline $\begin{array}{l}\text { Atitude para Utilizar } \\
\text { (ATU) }\end{array}$ & $\begin{array}{l}\text { ATU1 - Gosto da ideia de poder participar em atividades que utilizam wikis. } \\
\text { ATU2 - Se houver a possibilidade, pretendo utilizar wikis na minha aprendizagem. } \\
\text { ATU3 - Eu recomendo aos outros a utilização de wikis no processo EA. } \\
\text { ATU4 - Globalmente, a minha atitude é favorável à utilização de wikis no processo EA. }\end{array}$ \\
\hline
\end{tabular}

Fonte: Dados própria pesquisa.

Finalmente, questionaram-se os alunos sobre se, no caso de ser possível, pretendiam utilizar a ferramenta para elaborar o trabalho que especificaram no wiki. Com base nos dados recolhidos, foi feita uma análise descritiva para avaliar o grau de motivação para a utilização de wikis por parte dos alunos no contexto da aprendizagem. Além disso, foram feitos testes de hipóteses (Mann-Whitney) para comparar o referido grau de motivação entre os alunos que pretendiam continuar a utilizar o Wiki TAGI na realização do trabalho e os que não o 
pretendiam fazer. Nessas análises, foi utilizado o Software IBM SPSS Statistics 19.

\section{Resultados e discussão}

Nesta seção, apresentam-se e analisam-se os resultados obtidos na fase 3 do estudo, salientando-se que os alunos, antes da experiência com o Wiki TAGI, nunca tinham editado um wiki. Verificou-se, ainda, que a experiência de edição foi bastante dinâmica, na medida em que os alunos interagiram dentro e fora dos seus grupos de trabalho, evidenciando, assim, motivação relativa a trabalhos de natureza colaborativa. Os alunos introduziram diversas informações relativas aos seus trabalhos de grupo, tendo-se verificado que alguns deles foram para além dos objetivos estabelecidos, estruturando o wiki e utilizando diversos formatos de textos, tabelas e imagens de forma a enriquecer o trabalho.
Em seguida, apresentam-se os resultados relativos à motivação dos alunos para a utilização de wikis no contexto de aprendizagem e a comparação dessa motivação entre os alunos que pretendiam e os que nao pretendiam continuar a utilizar essa ferramenta para fazer o trabalho da UC.

\section{Motivação dos alunos para a utilização de wikis}

Na tabela 1, abaixo, apresentam-se as frequências absolutas e as estatísticas de localização relativas às respostas obtidas nas principais indagações colocadas no questionário baseado no modelo TAM, ao qual responderam 47 alunos.

Os itens apresentados avaliam vários aspetos da motivação para o uso wikis na aprendizagem, em uma escala de 1 (não concordo nada) a 5 (concordo totalmente).

Tabela 1 - Frequências e estatísticas de localização relativas aos itens referentes à motivação dos alunos para a utilização de wikis

\begin{tabular}{|c|c|c|c|c|c|c|c|c|c|}
\hline & \multicolumn{5}{|c|}{ Grau de concordância } & \multirow[b]{2}{*}{ Total } & \multirow[b]{2}{*}{ Média } & \multirow[b]{2}{*}{ Média } & \multirow[b]{2}{*}{ Média } \\
\hline & 1 & 2 & 3 & 4 & 5 & & & & \\
\hline $\begin{array}{l}\text { PEOU1 - É necessário consultar frequentemente o guião de apoio/ícone de ajuda } \\
\text { para utilizar os wikis. }\end{array}$ & 8 & 19 & 15 & 3 & 0 & 45 & 2,3 & 2 & 2 \\
\hline PEOU2 - Os ícones e o texto associados aos wikis são de fácil compreensão. & 0 & 3 & 7 & 31 & 6 & 47 & 3,9 & 4 & 4 \\
\hline $\begin{array}{l}\text { PEOU3 - É fácil lembrar-me de como executar as tarefas relacionadas com a edição } \\
\text { dos wikis. }\end{array}$ & 0 & 2 & 16 & 20 & 9 & 47 & 3,8 & 4 & 4 \\
\hline PEOU4 - Fico confuso quando utilizo um wiki. & 19 & 25 & 3 & 0 & 0 & 47 & 1,7 & 2 & 2 \\
\hline PEOU5 - Cometo erros frequentemente quando utilizo um wiki. & 8 & 24 & 11 & 3 & 0 & 46 & 2,2 & 2 & 2 \\
\hline PEOU6 - Aprender a utilizar os wikis é fácil. & 0 & 0 & 9 & 27 & 11 & 47 & 4,0 & 4 & 4 \\
\hline PEOU7 - No geral, acho que os wikis são fáceis de utilizar. & 0 & 1 & 2 & 38 & 6 & 47 & 4,0 & 4 & 4 \\
\hline PU1 - A utilização de wikis melhora o resultado da minha aprendizagem. & 1 & 6 & 19 & 15 & 5 & 46 & 3,4 & 3 & 3 \\
\hline PU2 - A utilização de wikis permite-me organizar melhor o meu trabalho. & 2 & 4 & 14 & 21 & 5 & 46 & 3,5 & 4 & 4 \\
\hline PU3 - A utilização de wikis faz-me poupar tempo. & 3 & 5 & 18 & 18 & 3 & 47 & 3,3 & 3 & 3 e 4 \\
\hline $\begin{array}{l}\text { PU4 - A comunicação proporcionada pelos wikis permite-me aprender através dos: } \\
\text { conteúdos colocados pelos outros. }\end{array}$ & 0 & 1 & 18 & 19 & 9 & 47 & 3,8 & 4 & 4 \\
\hline $\begin{array}{l}\text { PU5 - Os wikis permitem fazer trabalhos de grupo sem depender da presença física } \\
\text { dos outros elementos do grupo. }\end{array}$ & 1 & 6 & 8 & 19 & 13 & 47 & 3,8 & 4 & 4 \\
\hline $\begin{array}{l}\text { PU6 - Os wikis permitem fazer trabalhos de grupo sem estar dependente dos horários: } \\
\text { dos outros elementos do grupo. }\end{array}$ & 1 & 4 & 9 & 20 & 13 & 47 & 3,9 & 4 & 4 \\
\hline PU7 - No geral, acho os wikis úteis para a minha aprendizagem. & 1 & 1 & 13 & 24 & 7 & 46 & 3,8 & 4 & 4 \\
\hline ATU1 - Gosto da ideia de poder participar em atividades que utilizam wikis. & 0 & 6 & 19 & 17 & 4 & 46 & 3,4 & 3 & 3 \\
\hline ATU2 - Se houver a possibilidade, pretendo de utilizar wikis na minha aprendizagem. & 0 & 5 & 18 & 18 & 4 & 45 & 3,5 & 3 & 3 e 4 \\
\hline ATU3 - Eu recomendo aos outros a utilização de wikis no processo EA. & 0 & 7 & 13 & 20 & 5 & 45 & 3,5 & 4 & 4 \\
\hline ATU4 - Globalmente, a minha atitude é favorável à utilização de wikis no processo E/A & 1 & 2 & 18 & 21 & 5 & 47 & 3,6 & 4 & 4 \\
\hline
\end{tabular}

Fonte: Dados da pesquisa. 
De um modo geral, verifica-se que os alunos se manifestaram de uma forma positiva quanto aos vários itens relacionados com a PEOU, PU e ATU dos Wikis no processo EA.

Os itens mais bem classificados pelos alunos referem-se à facilidade de utilização percebida, sendo que os itens PEOU2, PEOU3, PEOU6 e PEOU7 refletem diretamente a facilidade de utilização da tenologia wiki. Verificou-se que entre $62 \%$ e $94 \%$ dos alunos atribuíram uma classificação de 4 ou 5. Os itens PEOU1, PEOU4 e PEOU5 apresentam níveis baixos, sendo, no entanto, as respostas igualmente favoráveis, uma vez que as questões foram colocadas usando frases na negativa, verificando-se que entre 57\% e 94\% dos alunos atribuíram os níveis 1 ou 2.

Relativamente aos itens que avaliam a utilidade percebida, registra-se que os mais classificados pelos alunos são os PU2, PU4, PU5, PU6 e PU7, verificando-se que entre 57\% e 70\% dos alunos lhes atribuíram os níveis 4 ou 5. Em particular, o PU4 é um dos itens com uma dispersão menor (apenas 2\% dos alunos atribuíram o nível 1 ou 2), revelando que, de uma forma geral, os alunos consideram que o fato de "A comunicação proporcionada pela utilização de wikis promover a aprendizagem através dos conteúdos colocados pelos outros" é uma das características mais úteis dessa tecnologia. A classificação favorável dos itens PU5, "Os wikis permitem fazer trabalhos de grupo sem depender da presença física dos colegas”, e PU6, "Os wikis permitem fazer trabalhos de grupo sem estar dependente dos horários dos outros elementos do grupo", confirmam os resultados do estudo de Oner (2009).

Nota-se, ainda, que os alunos não classificaram tão favoravelmente a melhoria obtida no resultado da aprendizagem (PU1) e o fato de pouparem tempo na sua realização (PU3).

Relativamente aos itens que avaliam a atitude para utilizar, verifica-se, que "Eu recomendo aos outros a utilização de wikis no processo EA" (ATU3) e "Globalmente, a minha atitude é favorável à utilização de wikis no processo EA" (ATU4), são classificados por cerca de 55\% dos alunos com os níveis 4 ou 5. Relativamente às questões "Gosto da ideia de poder participar em atividades que utilizam wikis" (ATU1) e "Se houver a possibilidade, pretendo utilizar wikis na minha aprendizagem" (ATU2), foram classificados por cerca de 40\% dos alunos no nível 3 e 46\% e $48 \%$, respetivamente, nos níveis 4 ou 5 . Esse resultado, menos favorável, pode ser explicado pelo receio de alguns alunos em expor o trabalho que estavam desenvolvendo, seja pela eventual possibilidade de haver colegas que pudessem aproveitar as suas ideias. 0 fato de qualquer pessoa poder modificar e/ou apagar os conteúdos que outra editou pode também ter influenciado as respostas a essa questão, como atesta o comentário de um dos entrevistados "[...] qualquer pessoa pode modificar o que escrevi, podendo apagar coisas importantes”.

Relativamente à utilização de wikis para a elaboração do trabalho de avaliação da UC, $67 \%$ dos alunos responderam que pretendem fazer uso da ferramenta.

Verificou-se, através dos resultados do questionário, que, de um modo geral, os alunos ficaram satisfeitos em relação à facilidade, à utilidade e à atitude para utilização dos wikis no processo EA.

\section{Comparação da motivação para utilizar wikis entre dois grupos de alunos}

Com o objetivo de verificar se o grau de concordância atribuído a cada um dos itens do questionário respondido pelos alunos que pretendem elaborar o trabalho utilizando o wiki é superior ao grau de concordância dos que não pretendem fazer uso da tecnologia, foram efetuados testes Mann-Whitney unilaterais, com nível de significância de 5\%.

0 teste de Mann-Whitney tem caráter não-paramétrico, alternativo ao teste de Student, em que são comparadas duas amostras independentes e cuja hipótese nula corresponde 
à situação em que as duas amostras provêm de populações com a mesma distribuição (ou da mesma população) (MURTEIRA, 1990). No caso de tal hipótese não ser rejeitada, admitem-se que as distribuições das duas populações são iguais.

Como se pode verificar na tabela 2, relativamente aos itens que avaliam a facilidade de utilização percebida, as distribuições dos dois grupos analisados não se revelaram significativamente diferentes.

Nos itens que avaliam a utilidade percebida e a atitude para utilizar, pode-se observar que as distribuições relativas aos dois grupos para cada item são significativamente diferentes na maioria dos casos - $71,4 \%$ do total dos itens no caso da utilidade uercebida e 50\% no caso da utitude para utilizar, sendo os itens PU2, PU4, ATU1 e ATU4, os que não apresentam diferenças significativas.

Realça-se que, relativamente ao item PU4, o fato de a afırmação que o traduz revelar a própria natureza da ferramenta, pode explicar a sua maior pontuação e a sua interiorização, de igual forma, por parte dos alunos dos dois grupos.

Tabela 2 - Testes Mann-Whitney relativos à comparação, entre os dois grupos de alunos, dos itens referentes à motivação para a utilização de wikis

\begin{tabular}{|c|c|c|c|}
\hline & Não & $\operatorname{Sim}$ & P-value \\
\hline PEOU1 - É necessário consultar frequentemente o guião de apoio/ícone de ajuda para utilizar os wikis. & 15 & $29 \vdots$ & 0,241 \\
\hline PEOU2 - Os ícones e o texto associados aos wikis são de fácil compreensão. & 15 & $31 \vdots$ & 0,188 \\
\hline PEOU3 - É fácil lembrar-me de como executar as tarefas relacionadas com a edição dos wikis. & 15 & $31 \vdots$ & 0,195 \\
\hline PEOU4 - Fico confuso quando utilizo um wiki. & 15 & $31 \vdots$ & 0,529 \\
\hline PEOU5 - Cometo erros frequentemente quando utilizo um wiki. & 15 & $30 \vdots$ & 0,084 \\
\hline PEOU6 - Aprender a utilizar os wikis é fácil. & 15 & $31 \vdots$ & 0,124 \\
\hline PEOU7 - No geral, acho que os wikis são fáceis de utilizar. & 15 & $31 \vdots$ & 0,289 \\
\hline PU1 - A utilização de wikis melhora o resultado da minha aprendizagem. & 15 & 30 & 0,002 \\
\hline PU2 - A utilização de wikis permite-me organizar melhor o meu trabalho. & 15 & $30 \vdots$ & 0,236 \\
\hline PU3 - A utilização de wikis faz-me poupar tempo. & 15 & $31 \vdots$ & 0,046 \\
\hline PU4 - A comunicação proporcionada pelos wikis permite-me aprender através dos conteúdos colocados pelos outros. & 15 & $31 \vdots$ & 0,381 \\
\hline PU5 - Os wikis permitem fazer trabalhos de grupo sem depender da presença física dos outros elementos do grupo. & 15 & $31 \vdots$ & 0,044 \\
\hline PU6 - Os wikis permitem fazer trabalhos de grupo sem estar dependente dos horários dos outros elementos do grupo. & 15 & $31 \vdots$ & 0,033 \\
\hline PU7 - No geral, acho os wikis úteis para a minha aprendizagem. & 15 & $30 \vdots$ & 0,004 \\
\hline ATU1 - Gosto da ideia de poder participar em atividades que utilizam wikis. & 15 & $30 \vdots$ & 0,162 \\
\hline ATU2 - Se houver a possibilidade, pretendo utilizar wikis na minha aprendizagem. & 15 & $29 \vdots$ & 0,021 \\
\hline ATU3 - Eu recomendo aos outros a utilização de wikis no processo E/A. & 15 & $29 \vdots \vdots$ & 0,021 \\
\hline ATU4 - Globalmente, a minha atitude é favorável à utilização de wikis no processo E/A. & 15 & $31 \vdots$ & 0,136 \\
\hline
\end{tabular}

Fonte: dados da pesquisa.

Nos itens ATU1 e ATU4, a não existência de diferenças entre as distribuições, pode ser explicada pela satisfação dos alunos relativamente a essa experiência e por sua atitude positiva face à ferramenta de uma forma global.

A figura 1 representa os diagramas tipo caixa do grau de concordância atribuído aos itens para os quais as diferenças encontradas foram estatisticamente significativas (PU1, PU3, PU5, PU6, PU7, ATU2 e ATU3) para os dois grupos (os alunos que pretendem elaborar o trabalho de avaliação com o wiki e os que não pretendem fazer uso da ferramenta). 
Figura 2 - Diagramas paralelos que representam o grau de concordância de cada item para os alunos que não pretendem elaborar o trabalho de avaliação utilizando o wiki (Não) e para os que planejam usar a ferramenta (Sim)

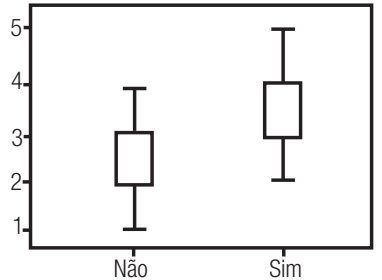

PU1- A utilização de Wikis melhora 0 resultado da minha aprendizagem.

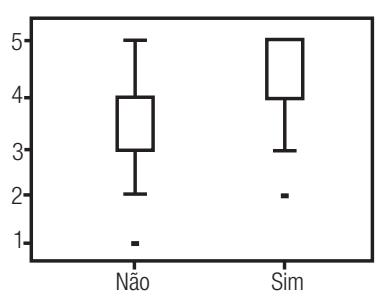

PU6- Os wikis permitem fazer trabalhos de grupo sem estar dependente dos horários dos outros elementos do grupo.

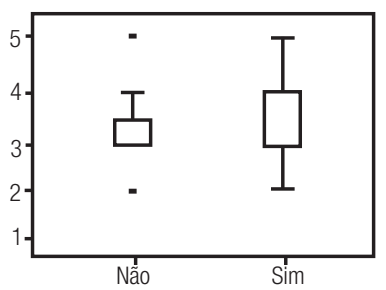

ATU2- Se houver a possibilidade de utilizar wikis úteis na minha aprendizagem, prendendo-o fazer.

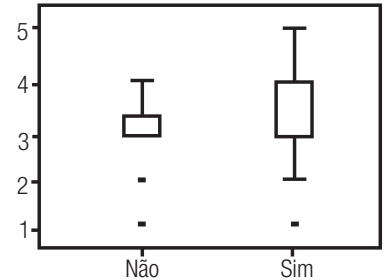

PU3- A utilização de Wikis me faz poupar tempo.

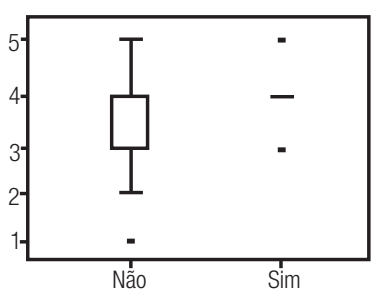

PU7- No geral, acho os wikis úteis para minha aprendizagem.

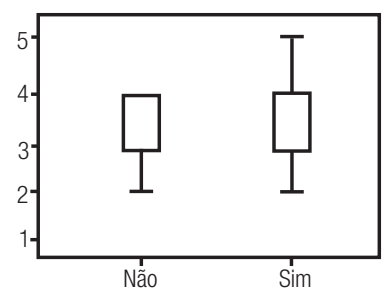

ATU3- Eu recomendo aos outros a utilização de wikis no processo de E/A.

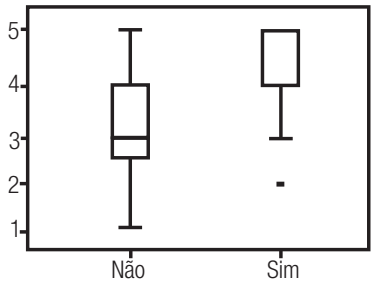

PU5- Os wikis permitem fazer trabalhos de grupo sem depender da presença física dos outros elementos do grupo.

Fonte: dados da própria pesquisa.

$\mathrm{Na}$ figura 2, pode-se verificar que, embora para todos os itens a distribuição das respostas se situe em níveis mais altos para o grupo dos alunos que pretende elaborar o trabalho utilizando o wiki em comparação com o grupo que não planeja fazer uso da ferramenta, salientam-se os casos em que essas diferenças são maiores - PU1 ("A utilização de wikis melhora o resultado da minha aprendizagem") e PU7 ("No geral, acho os wikis úteis para a minha aprendizagem”). Note-se que tais diferenças podem ser decorrência do fato de os alunos que pretendem utilizar a ferramenta no curto prazo terem uma maior crença nos benefícios que o uso dessa tecnologia pode proporcionar à sua aprendizagem individual.

\section{Conclusões}

0 wiki é uma tecnologia Web 2.0 que, segundo diversos estudos, apresenta inúmeras vantagens quando utilizada no contexto de ensino-aprendizagem (EA), permitindo a seus atores a troca de experiências através da edição, organização e partilha de conteúdos. 0 presente trabalho teve como objetivo avaliar a motivação da utilização dessa ferramenta por parte de um grupo de alunos da área tecnológica, no 
contexto de uma Unidade Curricular (UC) no ensino superior, e compará-la entre os alunos que pretendem usar a ferramenta para realizar um determinado trabalho de grupo com os que não pretendem fazer uso dessa tecnologia.

Verificou-se que, embora nunca tivessem editado um wiki antes da experiência levada a cabo no âmbito deste projeto, a grande maioria dos alunos não sentiu dificuldades na sua utilização, considerando a ferramenta útil para a sua aprendizagem.

Em particular, os dados revelaram uma postura positiva por parte dos entrevistado quando questionados a respeito da otivação para utilizar wikis no processo E/A. Os itens relativos à facilidade de utilização percebida foram, de forma geral, os mais bem classificados. Os itens relativos à utilidade percebida mais bem classificados foram: (i) a promoção da aprendizagem através dos conteúdos colocados pelos outros; (ii) a não dependência da presença física nem dos horários de todos para realizar trabalhos de grupo, e (iii) a utilidade geral da ferramenta.

Os itens relativos à atitude para utilizar foram, no geral, pontuados com níveis mais baixos do que os restantes, o que pode estar associado ao receio, manifestado por alguns alunos, de expor o trabalho que estavam desenvolvendo perante colegas que enfrentavam o mesmo desafio. 0 fato de qualquer pessoa poder modificar e/ou apagar os conteúdos que outra pessoa editou pode, também, ter influenciado as respostas a essas questões. Desta forma, considera-se a eventualidade da existência de inibição da utilização de um espaço aberto como os wikis para partilha de conteúdos que, pela sua natureza, possam ser alvo de cópia de ideias. Deverá, portanto, haver cuidado na seleção, por parte dos professores, do tipo de atividades desenvolvidas com recurso aos wikis e sugere-se a organização e a estruturação em seções do wiki e o estabelecimento, de uma forma clara, dos objetivos das atividades e de regras de compromisso com os alunos.

Adicionalmente, os resultados revelaram que, no que diz respeito à facilidade de utilização percebida, não se verificou a existência de diferenças significativas entre o grupo de alunos que tem a intenção de elaborar o trabalho de avaliação com o wiki e o grupo de alunos que não o pretende fazer esse uso. Já nos itens que avaliam a utilidade percebida e a atitude para utilizar, pode-se observar que as distribuições relativas aos dois grupos para cada item são significativamente diferentes na maioria dos casos - 71,4\% do total dos itens para a utilidade percebida e 50\% para atitude para utilizar.

Cabe destacar que este estudo foi levado a cabo com um grupo de alunos da área tecnológica, com conhecimentos sólidos na linguagem HTML, estando, portanto, em uma posição relativamente favorável à aprendizagem na edição de wikis, o que pode ter tido influência nos resultados. Dessa forma, pretende-se, como trabalho futuro, expandir a investigação a uma população de alunos de outras áreas menos tecnológicas e sem conhecimentos prévios de linguagem de edição wikis. Com isso, buscase comparar os resultados da futura pesquisa com os aqui apresentados e validar, também, a aceitação da tecnologia wiki. Pretende-se, igualmente, promover e divulgar esse meio de divulgação de conteúdos e de conhecimentos junto à comunidade acadêmica no âmbito de algumas atividades pedagógicas, quando essas justificarem a partilha de conhecimentos. 


\section{Referências}

CHAO, Joseph. Student project collaboration using wikis. 20th Conference on Software Engineering Education \& Training, CSEET ‘07, p. 255-261, 2007.

CHU, Samuel Kai-Wah. Twiki for knowledge building and management. Online Information Review, v. 32, n. 6, p. 745-758, 2008.

COLE, Melissa. Using wiki technology to support student engagement: lessons from the trenches. Computers \& Education, v. 52 , n. 1, p. 141-146, 2009.

COSTA, Carolina Simões da; TEIXEIRA, Leonor; ALVELOS, Helena. Web 2.0 no apoio ao processo ensino/aprendizagem: estudo exploratório no ensino superior, CAPSI' 11, Lisboa, 2011.

COUTINHO, Clara Maria Pereira; JUNIOR, João Batista Bottentuit. Collaborative learning using wiki: a pilot study with master students in educational technology in Portugal. World Conference on Educational Multimédia, Hypermedia e Telecommunications, ED-MEDIA'07, Vancouver, Canadá, p. 1786-1791, 2007.

CRESS, Ulrike; KIMMERLE, Joachim. A systemic and cognitive view on collaborative knowledge building with wikis. International Journal of Computer-Supported Collaborative Learning, v. 3, n. 2, p. 105-122, 2008.

CUBRIC, Marija. Wiki-based process framework for blended learning. WikiSym'07, 2007.

DAVIS, Fred. A technology acceptance model for empirically testing new end-user information systems: theory and results. (Doctoral dissertation). MIT Sloan School of Management, MIT Sloan School of Management, 1986.

. Perceived usefulness, perceived ease of use, and user acceptance of information technology. MIS Quarterly, v. 13, n. 3, p. 319-340, 1989.

; BAGOZZI, Richard; WARSHAW, Paul. User acceptance of computer technology: a comparison of two theoretical models. Management science, v. 35, n. 8, p. 982-1003, 1989.

DE PEDRO et al. Writing documents collaboratively in higher education using traditional vs. wiki methodology (I): qualitative results from a 2-year project study. 4th International Congress of University Teaching and Innovation, 2006.

DUFFY, Peter; BRUNS, Axel. The use of blogs, and RSS in education: A Conversation of Possibilities. Online Learning and Teaching, Brisbane, p.31-38, 2006.

FOORD, Dave. The stolen principle for using wikis educationally, 2007. Disponível em: <http://www.a6training.co.uk/ resources/STOLENticksheet.doc>. Acesso em: 13 jun. 2012.

FORTE, Andrea; BRUCKMAN, Amy. Constructing text: wiki as a toolkit for (collaborative?) learning. International symposium on wikis. Montreal, Quebec, Canadá, ACM, p. 31-42, 2007.

FRANKLIN, Tom; HARMELEN, Mark von. Web 2.0 for content for learning and teaching in higher education. Report on JISC Publications, 2007.

GOKCEARSLAN, Sahin; OZCAN, Seher. Place of wikis in learning and teaching process. Procedia Social and Behavioral Sciences, v. 28, n. 0, p. $481-485,2011$.

GRACE, Tay Pei Lyn. Wikis as a knowledge management tool. Journal of Knowledge Management, v. 13, n. 4, p. 64-74, 2009.

GROSSECK, Gabriela. To use or not to use web 2.0 in higher education? Procedia-Social and Behavioral Sciences, v. 1, n. 1, p. 478-482, 2009. 
GUO, Zixiu; STEVENS, Kenneth John. Factors influencing perceived usefulness of wikis for group collaborative learning by first year students. Australasian Journal of Educational Technology, v. 27, n. 2, p. 221-242, 2011.

HUANG, Wenhao David; YOO, Sun Joo. Correlations between usage and technology acceptance of Web 2.0 applications. AECT 2010: Cyber-change: learning in our connected world, Anaheim, CA, 2010.

IT-USER SERVICES. Wikis in higher education. An exploratory report about the value of wikis in higher education, from a faculty perspective. University of Delaware, 2008.

KANE, Gerald; FICHMAN, Robert. The shoemaker's children: using wikis for information systems teaching, research and publication. MIS Quarterly, v. 33, n. 1, p. 1-17, 2009.

KEAR, Karen; WOODTHORPE, John; ROBERTSON, Sandy; HUTCHISON, Mike. From forums to wikis: perspectives on tools for collaboration. The Internet and Higher Education, v. 13, n. 4, p. 218-225, 2010.

KWON, Ohbyung; WEN, Yixing. An empirical study of the factors affecting social network service use. Computers in Human Behavior, v. 26, n. 2, p. 254-263, 2010.

LAI, Yiu Chi; NG, Eugenia. Using wikis to develop student teachers' learning, teaching, and assessment capabilities. The Internet and Higher Education, v. 14, n. 1, p. 15-26, 2011.

MEI-YING, Wu; et al. A study of web 2.0 website usage behavior using TAM 2. Asia-Pacific Services Computing Conference, APSCC '08, IEEE, p. 1477-1482, 2008.

MEYER, Katrina. A comparison of web 2.0 tools in a doctoral course. The internet and higher education, v. 13, n. 4, p. 226-232, 2010.

MURTEIRA, Bento José Ferreira. Probabilidades e estatística. 2. ed. Lisboa: McGraw-Hill, 1990.

ÖNER, Diler. Pres-service teachers' experiences with wiki: challenges of asynchronous collaboration. Proceedings of the 5th international symposium on wikis and open collaboration. Orlando, Florida: ACM, p. 1-2, 2009.

PIFARRÉ, Manoli; STAARMAN, Judith Kleine. Wiki-supported collaborative learning in primary education: how a dialogic space is created for thinking together. International Journal of Computer-Supported Collaborative Learning, v. 6, n. 2 , p. 187-205, 2011.

RAITMAN, Ruth; AUGAR, Naomi; ZHOU, Wanlei. Employing wikis for online collaboration in the e-learning environment: case study. Third International Conference on Information Technology and Applications, ICITA'05, p. 142-146, 2005.

SU, Feng; BEAUMONT, Chris. Evaluating the use of a wiki for collaborative learning. Innovations in Education and Teaching International, v. 47, n. 4, p. 417-431, 2010.

ULRICH, Jana; KARVONEN, Meagan. Faculty instructional attitudes, interest, and intention: predictors of web 2.0 use in online courses. The Internet and Higher Education, v. 14, n. 4, p. 207-216, 2011.

VENKATESH, Viswanath; MORRIS, Michael; DAVIS, Gordon; DAVIS, Fred. User acceptance of information technology: toward a unified view. MIS Quarterly, v. 27, n. 3, p. 425-478, 2003.

WAN, Liyong; ZHAO, Chengling. Construction of a knowledge management framework based on web 2.0. Wireless communications, networking and mobile computing, WiCom'07, p. 5341-5344, 2007.

WESLEY, Shu; YU-HAO, Chuang. The behavior of wiki users. Social Behavior \& Personality: an International Journal, v. 39, n. 6 , p. 851-864, 2011.

WICHMANN, A. Preparing wikis for educational settings: the role of discussion board use in wiki-based writing. WikiSym'12, 2012. 
YANG, Chayan; HSU, Yi-Chun; TAN, Syuanti. Predicting the determinants of users' intentions for using YouTube to share video: moderating gender effects. Cyberpsychol Behav Soc Netw, v. 13, n. 2, p. 141-52, 2010.

ZEINSTEJER, Rita. The wiki revolution: a challenge to traditional education. TESL-EJ., v. 11. n. 4, 2008.

Recebido em: 03.12.2012.

Aprovado em: 26.03.2013.

Carolina Costa é licenciada em Ensino de Matemática e em Matemática Aplicada e Computação e mestre em Gestão de Informação pela Universidade de Aveiro, Portugal. Atualmente é estudante do Programa Doutoral em Engenharia e Gestão Industrial.

Helena Alvelos é licenciada em Engenharia Electrónica e de Telecomunicações pela Universidade de Aveiro, mestre em Gestão de Empresas (MBA) e doutora em Ciências da Engenharia pela Universidade do Porto, Portugal. Atualmente é professora auxiliar no Departamento de Economia, Gestão e Engenharia Industrial da Universidade de Aveiro e pesquisadora na unidade de investigação GOVCOPP.

Leonor Teixeira é licenciada em Engenharia e Gestão Industrial, mestre em Gestão de Informação e doutora em Gestão Industrial pela Universidade de Aveiro, Portugal. Atualmente é professora auxiliar no Departamento de Economia, Gestão e Engenharia Industrial da Universidade de Aveiro e pesquisadora na unidades de investigação IEETA e GOVCOPP. 